\title{
Changes in alpha activity reveal that social opinion modulates attention allocation during face processing
}

\author{
Evelien Heyselaar $^{\text {a, * }}$, Ali Mazaheri ${ }^{\text {b,c }}$, Peter Hagoort ${ }^{\text {a,d }}$, Katrien Segaert ${ }^{\text {b, }}$ \\ ${ }^{\text {a }}$ Max Planck Institute for Psycholinguistics, Nijmegen, The Netherlands \\ ${ }^{\mathrm{b}}$ School of Psychology, University of Birmingham, Birmingham, United Kingdom \\ ${ }^{\mathrm{c}}$ Centre for Human Brain Health, University of Birmingham, Birmingham, United Kingdom \\ ${ }^{\mathrm{d}}$ Donders Institute for Brain, Cognition and Behaviour, Nijmegen, The Netherlands
}

\section{A R T I C L E I N F O}

\section{Keywords:}

Social opinion

Face processing

Attention allocation

Alpha oscillation

Social facilitation

\begin{abstract}
A B S T R A C T
Participants' performance differs when conducting a task in the presence of a secondary individual, moreover the opinion the participant has of this individual also plays a role. Using EEG, we investigated how previous interactions with, and evaluations of, an avatar in virtual reality subsequently influenced attentional allocation to the face of that avatar. We focused on changes in the alpha activity as an index of attentional allocation. We found that the onset of an avatar's face whom the participant had developed a rapport with induced greater alpha suppression. This suggests greater attentional resources are allocated to the interacted-with avatars. The evaluative ratings of the avatar induced a U-shaped change in alpha suppression, such that participants paid most attention when the avatar was rated as average. These results suggest that attentional allocation is an important element of how behaviour is altered in the presence of a secondary individual and is modulated by our opinion of that individual.
\end{abstract}

\section{Introduction}

A commonly observed phenomenon in psychological research is that an individual's behaviour in a task is altered when conducted in the presence of another individual compared to when the task is done alone (Ringelmann, 1913). This effect is seen in a wide range of behaviours, for example participants eat more in the presence of someone else who is eating (Herman, 2015), participants' performance in cognitive tasks decreases for complex tasks in the presence of a secondary individual (Bond and Titus, 1983), and these changes occur even when interacting with human-like computers instead of actual secondary persons (Mandell et al., 2015). Although the effect of the presence of a secondary individual is not consistently positive or negative, there have been many behavioural studies establishing this phenomenon in different contexts. However, few have also investigated the mechanisms underlying the behaviour seen. Our study will be a step towards understanding how this process is implemented neurally.

In addition to the mere presence of a secondary individual, the opinion the participant has of this individual also influences the participants' subsequent behaviour (Heyselaar et al., 2017; Lott and Lott, 1961; Weatherholtz et al., 2014). One possible explanation for the phenomena discussed thus far involves the capture of attention by the secondary individual. Specifically, conducting a task in the presence of another person could cause one to divide their attention between the individual and the task, compared to when the task is done alone (for a review see Strauss, 2002). Additionally, if one finds the secondary individual more likeable, this could influence how much (or how little) attention is allocated to the secondary individual.

One way to investigate the neural processes related to attention is through electroencephalography (EEG), a non-invasive neuroimaging technique that measures the electrical potential generated by neurons. The EEG signal contains oscillatory activity in distinct frequency bands that have been found to map on to different facets of cognition (Siegel et al., 2012). Alpha activity, an oscillation occurring at a frequency of $10 \mathrm{~Hz}$, has been suggested to play a pivotal role in attention (Foxe et al., 1998; Mazaheri and Jensen, 2010). According to this framework, the suppression of alpha activity relates to the degree of cortical activation whereas an increase in alpha activity relates to cortical inhibition. It is proposed that alpha activity is mostly driven by forward propagating dendritic currents, and modulated (i.e. inhibited) by GABAergic feedback from interneurons (see for review Mazaheri and Jensen, 2010). While the precise mechanism in which alpha activity exerts functional inhibition is

\footnotetext{
* Corresponding author. Max Planck Institute for Psycholinguistics, Wundlaan 1, 6525XD, Nijmegen, The Netherlands.

E-mail address: evelien.heyselaar@mpi.nl (E. Heyselaar).
} 
not fully understood, some recent work suggests that alpha oscillations exercise a strong inhibitory influence on both spike timing and firing rate of neural activity (Haegens et al., 2011; Mazaheri and Jensen, 2010). Recently, a more nuanced view of alpha activity has been proposed in which its modulation of power is not strictly reflecting inhibition of sensory input (Van Diepen and Mazaheri, 2017). Rather, here the absolute baseline level of alpha power in a sensory cortex reflects the default allocation of neural resources earmarked to that region for processing. Any changes in alpha power in that region due to task demands reflect not necessarily inhibition but the redistribution of resources to optimise task performance. For this study we specifically focus on alpha activity as a representation of attentional allocation towards the secondary individual and modulations in the degree of alpha power/attention as a function of the opinion participants have of this secondary individual.

For this study, participants interacted with and subsequently evaluated digital secondary individuals (hereafter "avatars"). We measured the EEG activity during the viewing of the face of the avatars prior to their interaction and evaluation in Virtual Reality as well as after. As a control we also presented the face of avatars participants did not interact with. This design insures that the visual stimulus (in this case the face of the avatars) is constant, allowing us to investigate modulations of neural activity brought about by the interaction with, and evaluation of, the avatars.

In sum, the first aim of our study is to determine whether viewing an avatar the participant has interacted with results in a different degree of attentional allocation (as indexed by changes in alpha activity) compared to viewing an avatar the participant has not interacted with. We will therefore provide neuroimaging evidence which may clarify why participant behaviour is different when conducting a task in the presence of a secondary individual. Our study is one of the first, to our knowledge, to provide evidence for what has thus far only been theorised. The second aim is to determine whether the amount of attention allocated (measured as modulations of the change in alpha activity post-versus pre-interaction) to the avatar varies as a function of the participants' opinion of that avatar, something that again has not been tested before. Thus here we endeavour to be one of the first to provide a neurobiological explanation for behaviour seen in social psychology and social psycholinguistics.

Methods

Subjects

30 native Dutch speakers ( 2 male, $\mathrm{M}_{\mathrm{Age}}: 21.53$ years, $\mathrm{SD}_{\text {Age }}: 2.60$ ) gave written informed consent prior to the experiment and were monetarily compensated for their participation. As the EEG cap had to fit underneath the virtual reality (VR) helmet, we were limited to testing participants with small head sizes $(58 \mathrm{~cm}$ diameter and below), restricting us to mostly female participants. It is possible that there are gender differences in the influence of social opinion on task performance but our data do not allow us to investigate this further.

\section{Procedure}

The participants were informed that there were three phases to the experiment, but at the beginning of the experiment, they only received detailed information about Phase 1. At the start of Phase 2 they were informed of the goal of the study. The entire procedure is summarized in Fig. 1.

Participants initially viewed 565 static photos, of which 400 were of the 4 avatars. The faces of the four avatars were all exactly the same, and hence to be able to discriminate between photos of them, they were given different shirt colours. For Phase 2, participants interacted with three of the four avatars in Virtual Reality. Here the avatars were animated such that each avatar had different facial expressions in terms of their smile habit, eyebrow movements, and blink rate (see Table 1 ). The animations of the facial expressions were consistent for the entirety of the virtual interaction. Participants were therefore able to form opinions about the three avatars based on these facial characteristics, an effect seen robustly in previous studies using the same avatars and same facial expressions (Heyselaar et al., 2017). After each interaction, participants were asked to evaluate the avatars. For Phase 3, the participants were again shown static pictures. The participant used the colour of the shirt to discriminate

A

Phase 1

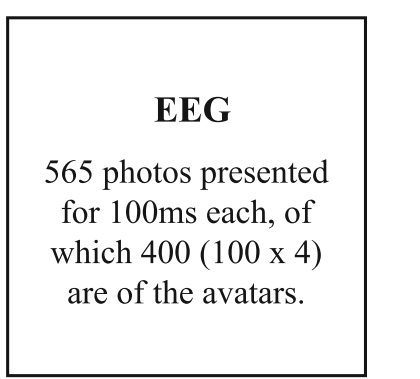

Phase 2

\section{VR}

10 min picture description task with 3 of the 4 avatars.

Avatars evaluated after each interaction.

B
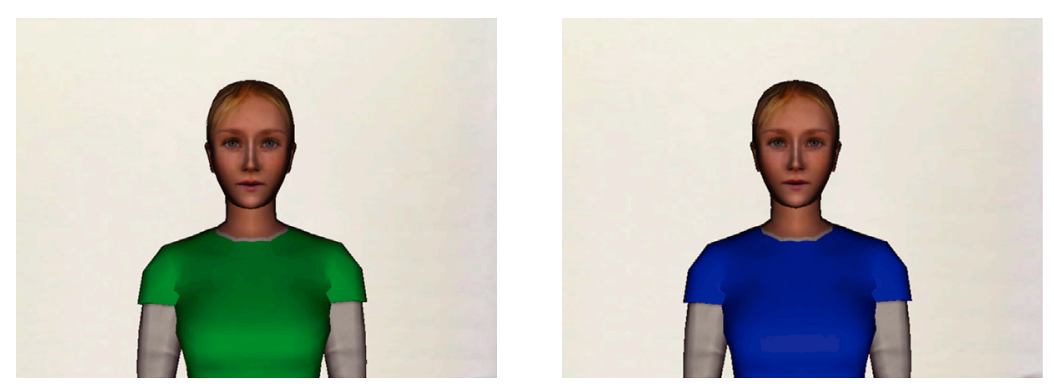

Fig. 1. A. Procedure B. Examples of two of the four avatars. Avatars were presented with green, blue, red, and yellow shirts. 
Table 1

Avatar facial expressions.

\begin{tabular}{llll}
\hline Avatar & Blink Duration $^{\mathrm{a}}$ & Smiling Habit & Eyebrow Habit \\
\hline 1 & No blink & No smile & No movement \\
2 & $0.1 \mathrm{~s}$ (Normal) & Dialogue-matched & Once every 3-5 s \\
3 & $0.1 \mathrm{~s}$ (Normal) & Dialogue-matched & Dialogue-matched \\
\hline
\end{tabular}

a Measured from the beginning of the closing movement to when the eye is fully open again.

between the avatars they had interacted with (and formed an opinion of) and the ones they had not interacted with. Details of each phase are given below:

\section{Phase 1 - Picture Evaluation Task}

In Phase 1 participants viewed photos of avatars and filler pictures, with the aim to measure EEG responses of the participants to the avatar pictures before they had formed any opinion of these avatars. The participants were instructed to evaluate pictures as either "likeable" or "not likeable." Each trial started with the presentation of a fixation cross for a 400-600ms jittered interval. This was followed by the presentation of a picture in the center of the screen. Pictures were presented for $100 \mathrm{~ms}$, followed by a jittered interval of $2000-3000 \mathrm{~ms}$ before an evaluation screen was presented. Participants were asked to indicate, via a button press, whether they liked or disliked the picture they just saw. The location of the options (left or right for "likeable") was randomized between participants.

Phase 1 consisted of 565 pictures. 400 of these pictures were of the avatars (100 repetitions of each avatar); the avatars are described below (Materials - Avatars). The remaining 165 were pictures selected from the pictures described below (Materials - Picture Evaluation Task). Picture order was randomized per participant.

\section{Phase 2 - Picture Description Task}

Participants interacted with three avatars for $10 \mathrm{~min}$ each in the virtual environment (these avatars are hereafter referred to as "Interacted-with avatars"). The EEG cap remained on in Phase 2, but no activity was recorded. The fourth avatar was not shown and not interacted with and therefore functioned as a control (hereafter "Non-interacted-with avatars"): in other words, by comparing the Interacted-with to the Noninteracted-with avatars, we can determine what the effects of interaction are. The shirt colour of all the avatars (both Interacted-with and Noninteracted-with) was pseudo-randomized across participants.

Participants at this point were informed of the goal of the study, to ensure they paid as much attention to the relevant characteristics of the avatar as possible.

The participant and the avatar would alternate in describing picture cards to each other. If the listener saw the card described by their partner as one of the cards in their spread they would select it, causing it to be automatically replaced by a novel card. The listener would then become the speaker and pick a card to describe. This continued until 50 cards were described, after which the headset was removed and participants were asked to fill out a pen-and-paper questionnaire about the avatar. We favoured a pen-and-paper questionnaire instead of having the avatar ask the questions directly as previous research has shown that if the participant evaluates the avatar in the presence of said avatar, they rate them more favourably (Nass et al., 1999).

The questionnaire consisted of three 6-point Likert-scale questions asking to rate the avatar on perceived humanness, perceived strangeness, and quality of their facial expressions in relation to the other two avatars (taken from Heyselaar et al., 2017). After each avatar, the participants were allowed to change their ratings for previously viewed avatars. The order of the avatars was pseudo randomized across participants.

Phase 3 - Picture Evaluation Task

In Phase 3, we again recorded EEG activity while participants viewed photos of the four avatars mixed with filler pictures. We kept Phase 3 the same as Phase 1, using the same picture order, therefore any difference in the modulation of alpha activity induced by viewing pictures of the avatars between Phase 1 and Phase 3 would likely be due to the social interaction that occurred in VR in Phase 2. By comparing the Interactedwith Avatar to the Non-interacted-with Avatar we can control for any repetition effects as both are viewed in both Phases. Before EEG recordings in Phase 3 began, impedance for each electrode was checked and adjusted as necessary.

\section{Materials}

\section{Picture Evaluation Task (Phase 1 and Phase 3)}

165 pictures were taken from the Geneva Affective PicturE Database (GAPED; Dan-glauser \& Scherer, 2011). An equal number (55) belonged to the category positive, negative or neutrally-rated pictures, in terms of emotional valence. We attempted to ensure that the arousal rating was comparable between picture categories as much as possible. The average ratings for arousal were thus 30.47 (SD: 9.491), 29.52 (SD: 5.92), and 46.46 (SD: 7.01) for positive, neutral, and negatively rated pictures respectively.

We also included four avatar photos: the same picture of the avatar with a yellow, green, red or blue shirt.

\section{Avatars and virtual environment in Phase 2}

All avatars had the same exterior adapted from a stock avatar ("casual15_f_highpoly"; see Fig. 1B) produced by WorldViz (www.worldviz. com, Santa Barbara, CA). All the avatars' speech was pre-recorded by the same human female and played during appropriate sections of the experiment. The avatars' appearance suggested that she was a Caucasian female in her mid-twenties, which matched the age and ethnicity of the Dutch speaker who recorded her speech. We opted to use the same voice as we wanted to ensure that any difference in opinion was due to the facial expressions, and not the style of speech.

The three facial expressions used have been tested elsewhere and have been convincingly demonstrated to induce a wide spread, and significantly different, ratings with regard to perceived humanness, perceived strangeness, and quality of facial expression (Heyselaar et al., 2017). These three facial expressions involved combinations of subtle changes in blink rate, smiling, and eyebrow habits (Table 1). Facial expression choices were based on work done by Looser and Wheatley (2010) who have shown that perception of humanness is dependent on upper face movement.

Blinks happened once every $1-5 \mathrm{~s}$ to mimic normal blink rate. For versions with normal smiling and normal eyebrow habits we explicitly programmed when the avatar would smile and/or raise her eyebrows, such that it would coincide with the content of her speech. For example, the avatar would raise her eyebrows when asking a question and smile when she was enthusiastic. When not speaking, she would smile once every $5-10 s$ and raise her eyebrows once every $1-5$ s such that she would still differ from the no smile/no eyebrow version. All of these changes were extremely subtle to ensure that they can still be related to ecologically valid behavioural characteristics that one would encounter in the everyday world.

The virtual environment (VE) was a stock environment produced by WorldViz ("room.wrl") adapted to include a table with a wooden divider. We chose to have the cards displayed at the top of the divider so that the participants could see the cards while facing forward. This was done due to the weight of the head-mounted display (HMD), which would cause an uncomfortable strain on the back of the participants' heads when they face down. Having the participants face forward throughout the entire experiment distributes this weight more comfortably.

The experiment was programmed and run using WorldViz's Vizard software. Participants wore an NVIS nVisor SX60 HMD, which presented the VE at $1280 \times 1024$ resolution with a 60 -degree monocular field of view. Mounted on the HMD was a set of 8 reflective markers linked to a 
passive infrared DTrack 2 motion tracking system from ART Tracking, the data from which was used to update the participant's viewpoint as she moved her head. It is known that this type of headset can cause dizziness and nausea due to the exclusion of the participant's nose in the field of view (Whittinghill et al., 2015). However, as each interaction was quite short ( $\sim 5 \mathrm{~min}$ ), none of our participants reported feeling any nausea.

Additionally, a single reflective marker was taped onto the index finger of the participant's dominant hand. This marker was rendered as a white ball in the VE, such that participants knew the position of their finger at all times. Sounds in the VE, including the voice of the avatars, were rendered with a 24-channel WorldViz Ambisonic Auralizer System.

\section{Picture Description Task in Phase 2}

The pictures used in this task have been described elsewhere (Segaert et al., 2011; Menenti et al., 2011). Our stimulus pictures depicted 40 transitive events such as kissing, helping or strangling with the agent and patient of this action. Each event was depicted by a greyscale photo containing either one pair of adults or one pair of children. These pictures were used to elicit transitive sentences; for each picture speakers can either produce an active transitive sentence (e.g. the woman kisses the man) or a passive transitive sentence (e.g. the man is kissed by the woman).

We also included pictures depicting intransitive events such as running, singing, or bowing using one actor. The actor could be any of the actors used in the transitive stimulus pictures.

Each card consisted of one stimulus picture with the relevant verb printed underneath.

\section{Data analysis approach}

\section{Pre-processing}

EEG was recorded from 64 cap-mounted $\mathrm{Ag} / \mathrm{AgCl}$ electrodes (ActiCAP, Brainproducts). Horizontal eye movements were monitored by two electrodes placed at the outer left and right canthi. Vertical eye movements were monitored using an electrode placed below the left eye. In addition, electrodes were placed on the left and right mastoid bones. During EEG recording, all electrodes were referenced to the left mastoid. All impedances were kept below $10 \mathrm{k} \Omega$. Signals were recorded with a BrainAmp amplifier system, using a $150 \mathrm{~Hz}$ low-pass filter, a time constant of $10 \mathrm{~s}(0.016 \mathrm{~Hz})$, and a $500 \mathrm{~Hz}$ sampling rate. Signals were later rereferenced off-line to linked mastoids.

The pre-processing of the data was done using functions from EEGLAB (Delorme and Makeig, 2004) and the Fieldtrip software package (Oostenveld et al., 2011). Fieldtrip EEG epochs were locked to the onset of the picture and manually inspected for non-physiological artefacts. Ocular artifacts were removed using independent component analysis (infomax algorithm) incorporated as the default "runica" function in EEGLAB. $3.48 \%$ of trials were discarded due to artefacts.

\section{Time-frequency representations (TFR) of power}

Time-frequency representations (TFR) of power were calculated for each trial using sliding Hanning tapers having an adaptive time window of three cycles for each frequency of interest $(\Delta T=3 / f)$, utilizing the same approach used in previous studies e.g., Mazaheri et al. (2014) and van Diepen et al. (2015).

The classification of frequency bands in delta $(2-4 \mathrm{~Hz})$, theta (5-8 Hz), alpha $(8-14 \mathrm{~Hz})$, high alpha $(11-14 \mathrm{~Hz})$, and beta $(15-20 \mathrm{~Hz})$ for further analysis were based on prior literature (Hamel-Thibault et al., 2015; Kliegl et al., 2015; Klimesch, 1997; Lange et al., 2013; Shahin and Pitt, 2012).

Changes in the power of oscillatory activity induced by the onset of avatar faces were expressed in terms of change scores from baseline $\left(\Delta P_{t}\right)$ using the following formula: $\Delta P_{t}=\left(P_{t}-P_{r}\right) / P_{r}$, where $P_{r}$ was the mean power during the baseline period $150 \mathrm{~ms}-650 \mathrm{~ms}$ before the onset of the picture and $P_{\mathrm{t}}$ was the power at each specific time point.

\section{Statistical analysis}

\section{Correction for multiple comparisons}

We corrected for multiple-comparisons (multiple electrodes) by means of a non-parametric cluster level (over-electrodes) randomization routine (Maris and Oostenveld, 2007). In this procedure, for each contrast (e.g., Phase 3 versus Phase 1) first a two-tailed dependent $t$-test was computed for each individual electrode-time pairs for every single time point. Next, electrodes which passed the significance threshold at a $5 \%$ significance level were clustered by direction of effect and spatial proximity (i.e. how close the electrodes are). These clusters were averaged across the time that was significant. A Monte Carlo probability value was estimated for this cluster by randomly swapping the conditions within subjects and calculating the maximum cluster-level test statistic 1000 times. We have employed a similar procedure coded in the Fieldtrip toolbox in a number of previous studies (van Diepen et al., 2015; van Diepen et al., 2016; van Diepen and Mazaheri, 2017; Segaert et al., 2017).

\section{Mixed models}

The values extracted from the electrodes of interest (see Results) were analysed using a linear mixed effects model, using the lmer function of the lme4 package (version 1.1.9; Bates et al., 2012) in R (R Core Development Team, 2011). The dependent measure was the values extracted from the regions of interest. The repeated-measures nature of the data was modelled by including a per-participant and per-shirt-colour random adjustment to the fixed intercept ("random intercept"). We began with a full model (two-way interactions between each of the ratings) and then performed a step-wise "best-path" reduction procedure, removing interactions before main effects, to locate the simplest model that did not differ significantly from the full model in terms of variance explained. All ratings were centred before entry into the model. $P$ values were extracted using the Anova function from the car package (version 2.1.0; Fox and Weisberg, 2011) using Wald Chi-Square tests (Type III).

\section{Results}

All data were normalized as percent change from a baseline interval (150-650ms before picture onset) within participants to reduce the contribution of participants with large variance in the power estimates. To identify time windows of interest we compared the time-frequency data between Phase 3 and Phase 1 (Fig. 2A). These data represent changes in spectral power due to repetition effects, as the task was exactly the same between Phase 1 and Phase 3. A non-parametric clusterbased permutation analysis was used to determine time points of interest for delta, theta, alpha, and beta frequency bands. Time points of interest identified are listed in Table 2, and illustrated in Fig. 2B. Strictly speaking, one would need to correct for multiple comparisons since the analyses in delta, theta, and beta are not hypothesis driven but exploratory (so $\mathrm{p}<.016$ to be considered significant). If we apply this to our results, the delta and one theta cluster does not survive correction.

To control for low-level sensory-induced oscillatory changes in the EEG induced during the onset of pictures, we subtracted the EEG activity in Phase 1 from the EEG activity in Phase 3. This ensures that we are subtracting any EEG activity related to visual onset of the pictures (and performing the 'like/dislike' task) since this occurred in Phase 1 as well as in Phase 3.

\section{Interacted-with avatars induced greater alpha suppression than non- interacted-with avatars}

We set out to investigate whether oscillatory activity is modulated by the experience of interacting with and evaluating the traits (both occurring in Phase 2) of 3 of the 4 avatars. We limited our analysis to the time and frequency windows identified above. Although the contrasts are asymmetric (300 Interacted-with avatar trials compared to 100 Non- 
A

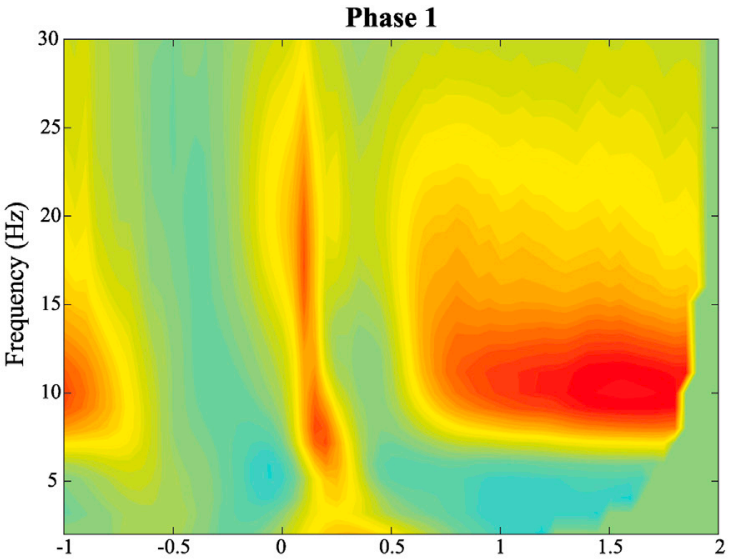

Phase 3

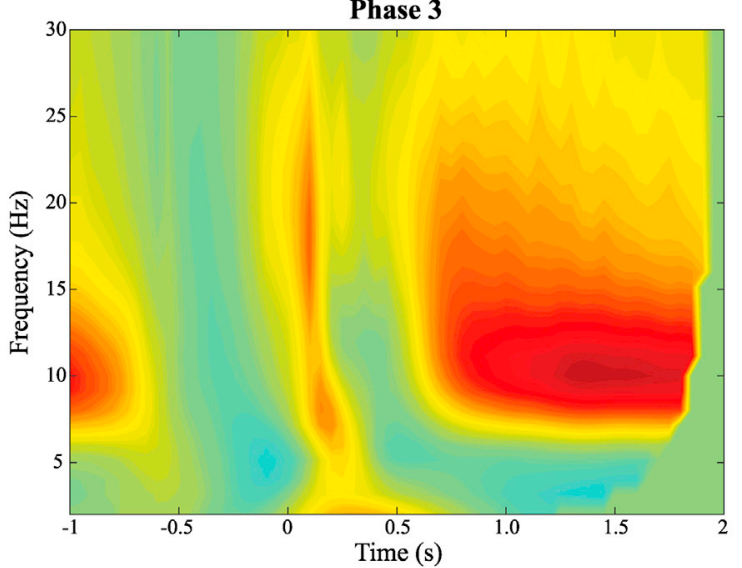

B
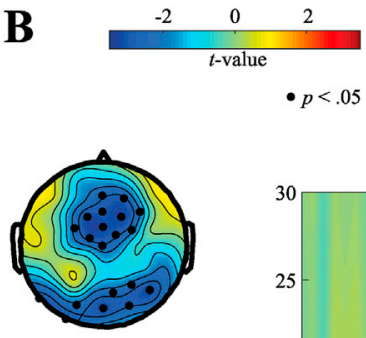

Alpha $(8-14 \mathrm{~Hz})$ $100-500 \mathrm{~ms}$

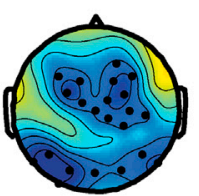

Theta $(5-8 \mathrm{~Hz})$ $100-350 \mathrm{~ms}$

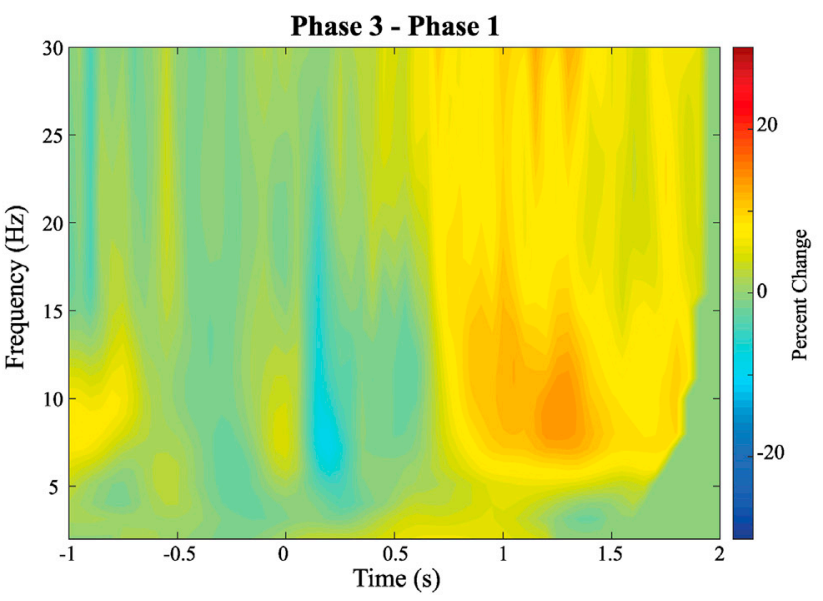

Beta $(15-20 \mathrm{~Hz})$ $700-1150 \mathrm{~ms}$

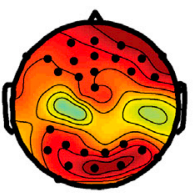

Alpha $(8-14 \mathrm{~Hz})$ $750-1500 \mathrm{~ms}$

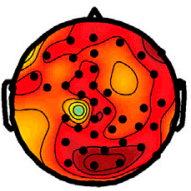

Theta $(5-8 \mathrm{~Hz})$ $700-1500 \mathrm{~ms}$

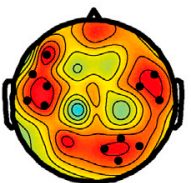

Delta $(2$ - $4 \mathrm{~Hz})$

$900-1200 \mathrm{~ms}$
Fig. 2. TFR of all conditions for all electrodes for A. Phase 1 and Phase 3 separately and $\mathrm{B}$. for Phase 3 minus Phase 1 . We used a non-parametric cluster based permutation test to identify time points of interest that were significantly different between Phase 3 and Phase 1 . These time intervals of interest (TOI) are used in the subsequent analysis. The top panel of section $\mathrm{B}$ shows the topography of the significant power changes in the different frequency bands. The electrodes which showed a significant different between Phase 3 and Phase 1 are marked with dots $(p<.05$, Monte Carlo estimated). TFRs are expressed as a percentage change from baseline $(-650 \mathrm{~ms}$ to $-150 \mathrm{~ms}$ before face onset). 
Table 2

Time points of significant difference in oscillatory power between Phase 3 and Phase 1 (Phase $3>$ Phase 1 ). $P$ values were obtained using Monte Carlo simulations (1000 iterations), corrected for multiple comparisons.

\begin{tabular}{lll}
\hline Frequency Range $(\mathrm{Hz})$ & Time Period $(\mathrm{ms})$ & $p$ value \\
\hline 2-4 (Delta) & $900-1200$ & .036 \\
5-8 (Theta) & $100-350$ & two clusters; $p<.020$ \\
& $700-1500$ & .003 \\
8-14 (Alpha) & $100-500$ & two clusters; $p<.047$ \\
& $750-1000$ & two clusters; $p<.006$ \\
15-20 (Beta) & $700-1150$ & .002 \\
\hline
\end{tabular}

interacted-with avatar trials), a post hoc analysis using 100 randomly drawn Interacted-with avatar trials revealed the same results as those reported below (see Supplementary Fig. 1).

The comparison of alpha power between Interacted-with and Noninteracted-with avatars revealed that for the Interacted-with avatars high alpha (11-14 Hz) was significantly more suppressed 400-450ms post-face onset for a cluster of parietal electrodes (Monte Carlo $p=.017$, corrected for multiple comparisons). Later alpha activity $(8-14 \mathrm{~Hz})$ between 750 and $1000 \mathrm{~ms}$ post-face onset (Monte Carlo $p=.050$, corrected for multiple comparisons) was also significantly more suppressed for Interacted-with avatars. There was also a significantly greater suppression of beta power $(15-20 \mathrm{~Hz}) 700-1000 \mathrm{~ms}$ post-face onset (Monte Carlo $p=.015$, corrected for multiple comparisons) for Interacted-with compared to Non-interacted-with avatars for a cluster of midline central electrodes. There were no significant effects found in any of the other time intervals of interest. The significant clusters are illustrated in Fig. 3.

\section{Amount of alpha suppression for the interacted-with avatars was modulated} by social opinion ratings

The increase in alpha suppression observed for Interacted-with avatars suggests greater attentional resources were allocated to them than to the Non-interacted-with avatars. The second aim of this study is to determine whether the alpha modulation (i.e. attention allocation) varied as a function of how the avatars were rated on a certain trait.

The purpose of having the participants interact with three different avatars was to ensure as wide a range of ratings per trait as possible. Previous research using these three avatars (Heyselaar et al., 2017) has shown that analysing the data per avatar instead of per trait results in an averaging out of any effects. This is due to individual differences: the impression Avatar 1 makes on one participant is not necessarily the same impression that same avatar makes on a different participant. An analysis per rating/trait thus provides a more accurate representation of any underlying effect (for more information, see Heyselaar et al., 2017).

Table 3 shows the spread of ratings for each of the three traits (perceived humanness, perceived strangeness, and quality of facial expression) where a higher rating represents a higher score in said trait (more human, more strange, higher quality of facial expression). Participants were asked after interacting with each avatar to rate said avatar on each of these traits in relation to the other two avatars. For this reason, participants were allowed to change their answers after having interacted with all three.

As predicted, we have a wide spread of ratings across most of the traits, with only the outer most ratings having 3 data points or less. Before using these data in any analysis, they were first trimmed to only include ratings with 7 or more data points. In other words, we removed rating 1 $(\mathrm{N}=1)$ and $6(\mathrm{~N}=2)$ for perceived humanness, rating $5(\mathrm{~N}=3)$ and 6 $(\mathrm{N}=0)$ for perceived strangeness, and rating $6(\mathrm{~N}=2)$ for quality of facial expression.

To determine if these ratings can modulate the activity shown in Fig. 3, we used the channels that showed significant activity as regions of interest. We extracted the average values for each participant for each avatar for the alpha, beta, and theta band frequency and time windows as specified above. The values for Phase 1 were then subtracted from the values for Phase 3 to control for any changes in activity related to the processing of photos. In the resulting dataset, the values for the Noninteracted-with avatars were then subtracted from the values for the Interacted-with avatars to control for the fact that the photos were viewed for a second time. The resulting values therefore capture any changes in activity due to how participants process the Interacted-with avatars. These resulting values were then entered into a mixed model along with the trimmed behavioural ratings for each of the three traits in Table 2.

The model for the picture-induced alpha suppression (400-450ms; $11-14 \mathrm{~Hz}$ ) showed a tendency for the Perceived Humanness rating to predict alpha activity $\left(\chi^{2}(1)=3.55, p=.059\right)$. Perceived Strangeness was in the best model, but showed no significant contribution $\left(\chi^{2}(1)=0.20\right.$, $p>.250)$. Fig. 4 shows the influence of Perceived Strangeness and Perceived Humanness on changes in alpha activity between Interactedwith and Non-interacted-with avatars.

Due to the quadratic shape of the effect of Perceived Strangeness on alpha activity, we re-ran the mixed effects model with Perceived Strangeness modelled as a quadratic term. The results of this model are summarized in Table 4. The quadratic term significantly predicted the alpha suppression $(p=.010)$. Our interpretation is that the perception of this trait modulates attention allocation (as indexed by the alpha suppression) such that avatars which are perceived as being very strange or not very strange draw the least amount of attention, whereas those that are perceived as being medially-strange draw the most amount of attention.

Even though our main interest was in modulations of alpha suppression, which represent modulations in attention allocation, as we found significant changes in beta power between the Interacted-with and Non-interacted-with avatars, we next also modelled these changes in the same way as described above.

The model for picture-induced beta suppression $(700-1000 \mathrm{~ms}$; $15-20 \mathrm{~Hz}$ ) showed a tendency for the Quality of Facial Expression ratings to predict beta activity. Beta oscillations are commonly correlated with imagined motor preparation (McFarland et al., 2000; Pfurtscheller et al., 1997) and hence these correlations may be indicators of what factors the participants use to rate the evaluated avatars in Phase 3. Beta values of the model showed that increased ratings of Quality of Facial Expression decreased $(\beta=-0.10)$ beta suppression in the Interacted-with compared to the Non-interacted-with avatar.

\section{Discussion}

It is well established in psychological science that the presence of a secondary individual influences a participants' behaviour on a task and, moreover, the opinion the participant has of this secondary individual also affects behaviour. We hypothesized that both phenomena can be explained by the capture of attention. In the current study we investigated this by examining changes in alpha suppression after participants had interacted with and evaluated digital individuals ("avatars"). The first aim of our study was therefore to determine whether viewing an avatar the participant has interacted with resulted in a different degree of alpha modulation (which we suggest is an index of attentional allocation) and we found that this was indeed the case. We also examined if the changes in alpha activity observed differed as a function of the ratings participants had given the secondary individual before we recorded their EEG activity and indeed found a relationship between evaluation ratings of the secondary individual and modulations in alpha suppression. We therefore conclude that attentional capture, as measured by changes in alpha activity, is an important step towards understanding how secondary individuals influence participants' behaviour.

We will go into the results of each of our two aims in detail. Our first aim was to determine whether attentional resources were differentially allocated when participants viewed faces of individuals they had just interacted with. To test this, participants viewed pictures of avatars before and after interacting with and evaluating them (Interacted-with 

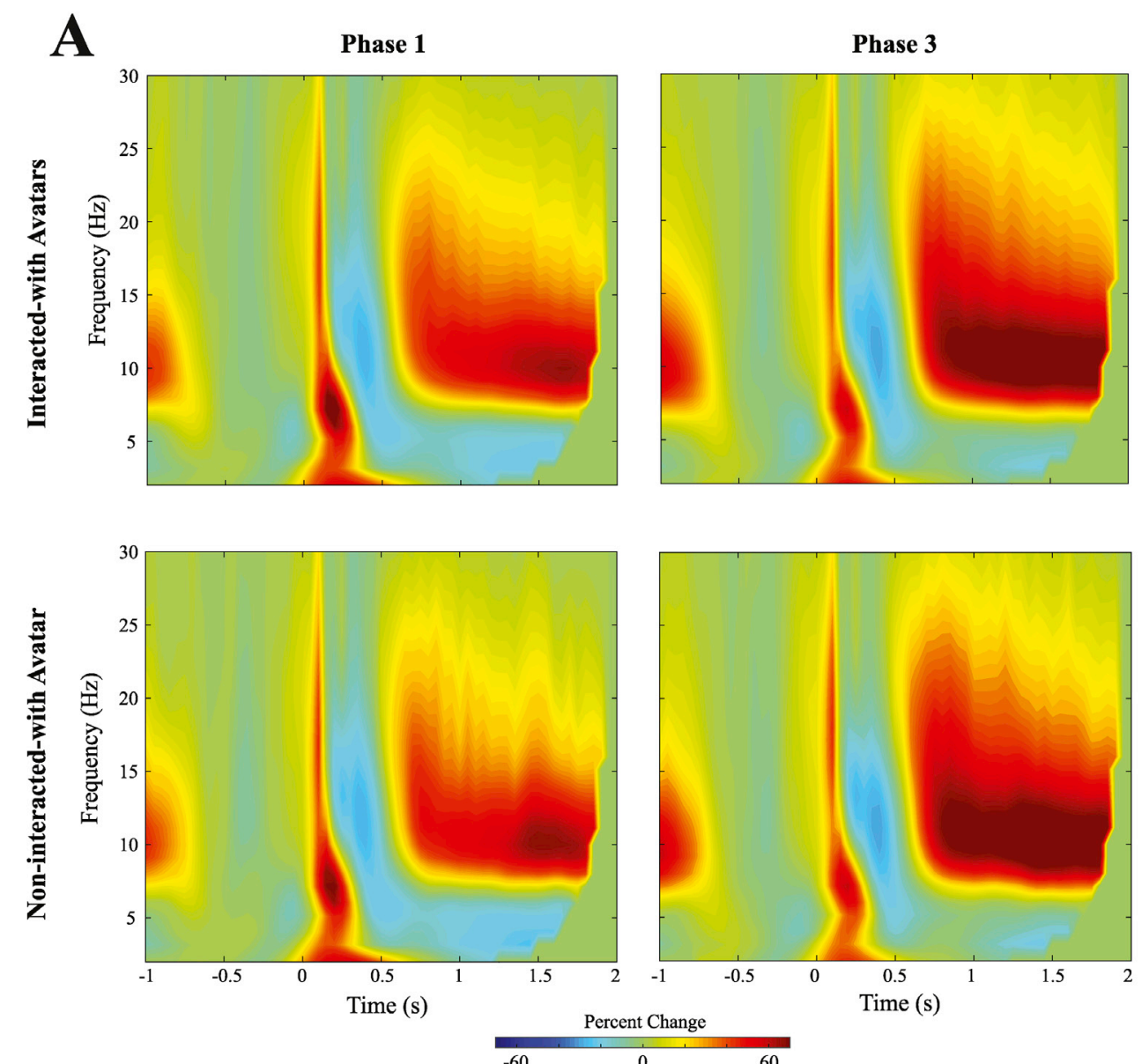

B
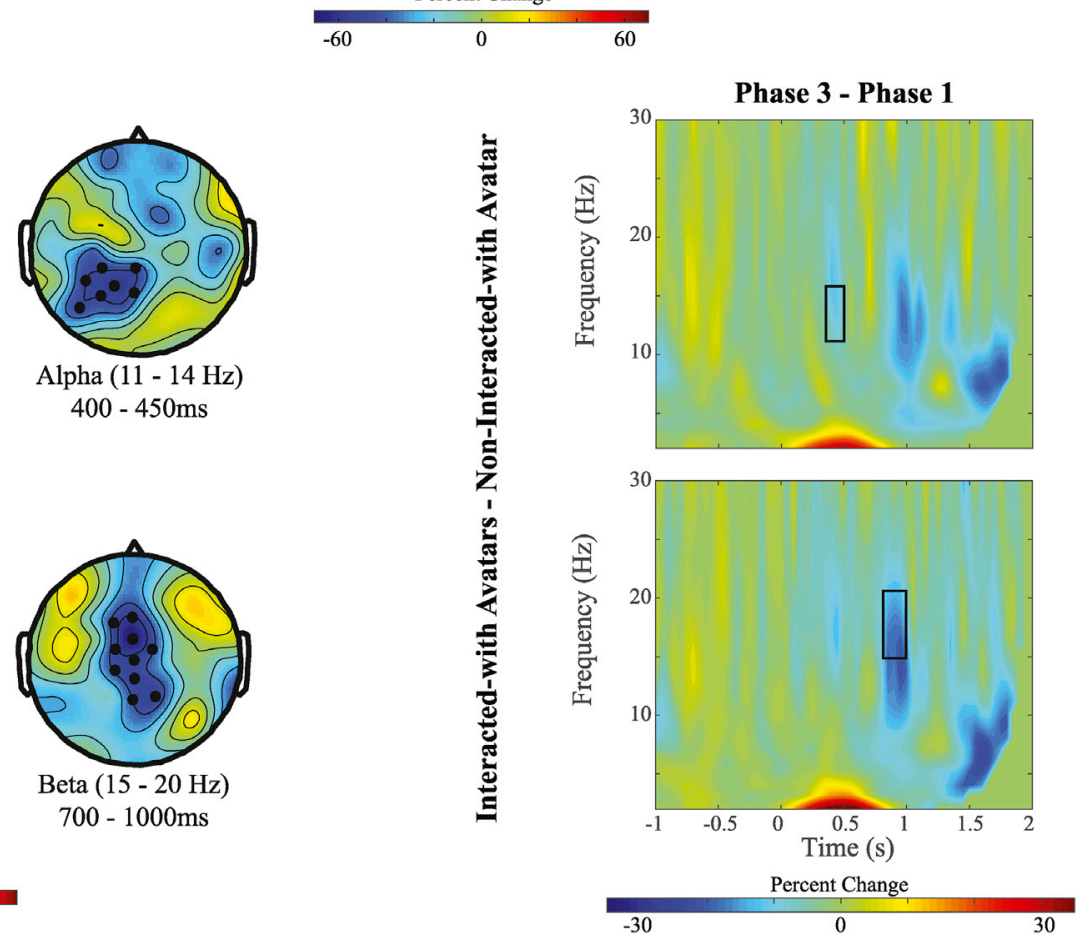

Fig. 3. Power difference between the Interacted-with and Non-interacted-with avatars A. The TFR of all electrodes for the Interacted-with and Non-interacted-with avatars for Phase 1 and Phase 3. B. The TFR for the cluster of electrodes showing a significant difference between Interacted-with avatars and Non-interacted-with avatars, after subtracting Phase 1 from Phase 3. This comparison reflects processing related only to having interacted with the avatar and evaluating their traits (both occurring in Phase 2). The topography contains the two significant clusters (marked with dots): $11-14 \mathrm{~Hz}$ (Alpha), 400-450ms post-face onset; and 15-20Hz (Beta), $700-1000 \mathrm{~ms}$ post-face onset. TFRs are expressed as a percentage change from baseline ( $-650 \mathrm{~ms}$ to $-150 \mathrm{~ms}$ before face onset). 
Table 3

Number of Ratings in Each Likert-Scale Trait. Three traits were tested (perceived humanness, perceived strangeness and quality of facial expressions). Higher ratings represent a higher score in said trait (more human, more strange, higher quality of facial expression).

\begin{tabular}{lllllll}
\hline \multicolumn{7}{l}{ Ratings } \\
Trait & 1 & 2 & 3 & 4 & 5 & 6 \\
\hline Perceived Humanness & 1 & 18 & 22 & 37 & 10 & 2 \\
Perceived Strangeness & 7 & 34 & 25 & 21 & 3 & 0 \\
Quality of Facial Expression & 9 & 25 & 21 & 24 & 9 & 2 \\
\hline
\end{tabular}

avatars). While viewing the avatar's faces, we recorded participants' EEG activity. The Non-interacted-with avatars appeared in both parts of the EEG experiment but were not interacted with. We initially identified four frequency bands of interest: delta, theta, alpha, and beta. Only the alpha and beta bands showed a significant difference when participants viewed faces of Interacted-with compared to Non-interacted-with avatars. We will go into the specifics of these two results below.

For the alpha band effect, we found that the Interacted-with avatars induced a greater amount of alpha suppression $400 \mathrm{~ms}$ after picture onset compared to the Non-interacted-with avatar. Alpha suppression occurred over the occipital parietal cortices with a high peak frequency $(11-14 \mathrm{~Hz})$, which has been previously associated with semantic processing demands (Klimesch, 1997). This suggests that pictures of the Interacted-with avatars have been allocated more resources for processing compared to the Non-interacted-with avatars. The left posterior parietal location of this cluster is suggestive of an effect indicating enhanced perceptual analysis of the input (De Cesarei and Codispoti, 2011).

For the beta band effect, we observed a greater amount of beta suppression 700ms after picture onset for the Interacted-with compared to the Non-interacted-with avatar. We hypothesize that this beta suppression is indicative of what factors influence the participant's motor response in the 'Like/Dislike' task that followed $2 \mathrm{~s}$ post stimulus onset (Pfurtscheller et al., 1997; McFarland et al., 2000). The central location of the significant clusters as opposed to the ipsi-/contra-lateral locations usually seen in the literature is most likely due to us randomizing the location of the Like and Dislike button between participants.

Our second aim was to determine whether the degree of pictureinduced alpha modulation for Interacted-with avatars differed as a function of the opinion the participant had of the secondary individual. All the avatars were exactly the same, except for the facial expressions, which we have shown in two previous studies to be enough to impact people's opinion of these avatars (Heyselaar et al., 2017; Heyselaar et al., 2015). However, as they were identical in appearance, we cannot rule out the possibility that they were perceived not as individuals but as the same avatar with varying moods. This does not change the interpretation of our results, as the participants had a different opinion of each avatar, even if it is just in a different mood. Therefore any effects this may have on alpha oscillations could be interpreted as an effect of opinion on alpha modulation.

The main finding for our second aim was the modulation of alpha suppression as a function of the perceived strangeness rating in the form of a U-shaped curve for the early alpha effect $(400-450 \mathrm{~ms})$. The mostand least-strangely rated avatars induced less alpha suppression for the Interacted-with compared to the Non-interacted-with avatars, whereas the medially-rated avatars elicited less alpha suppression for the Noninteracted-with compared to the Interacted-with avatars. This suggests that the perception of the most- and least-strangely rated avatars requires fewer resources compared to perception of the medially-rated avatars.

The results of the current study are consistent with previous literature looking at the effect of face likeability and distinctiveness on memory performance. Lin and colleagues (2011) had participants learn adjectives associated with faces and observed a U-shaped, quadratic curve such that participants had worse recall performance for the adjectives matched with faces rated as averagely-distinctive and best performance for adjectives presented with likeable and unlikeable faces. We can link this to our attention hypothesis: the effect on attention that we observe in our study could be due to more resources being required to retrieve information about the medially-rated avatar, as they were not remembered as well as the most- and least-strangely rated avatars.

The avatars we used in this study have been used before to elicit a difference in syntactic priming as a function of the strangeness rating (Heyselaar et al., 2017). Syntactic priming refers to the phenomenon

Table 4

Summary of best linear mixed model for changes in alpha activity between Interacted-with and Non-interacted-with avatars.

\begin{tabular}{llllll}
\hline & coefficient & $S E$ & $t$ value & $p$ value & \\
\hline Intercept & -0.41 & 0.17 & -2.49 & .010 & $*$ \\
Perceived Strangeness (quad.) & 0.10 & 0.04 & 2.56 & .010 & $*$ \\
Perceived Humanness (linear) & -0.21 & 0.12 & -1.78 & .075 &. \\
\hline
\end{tabular}

$\mathrm{N}=84$.
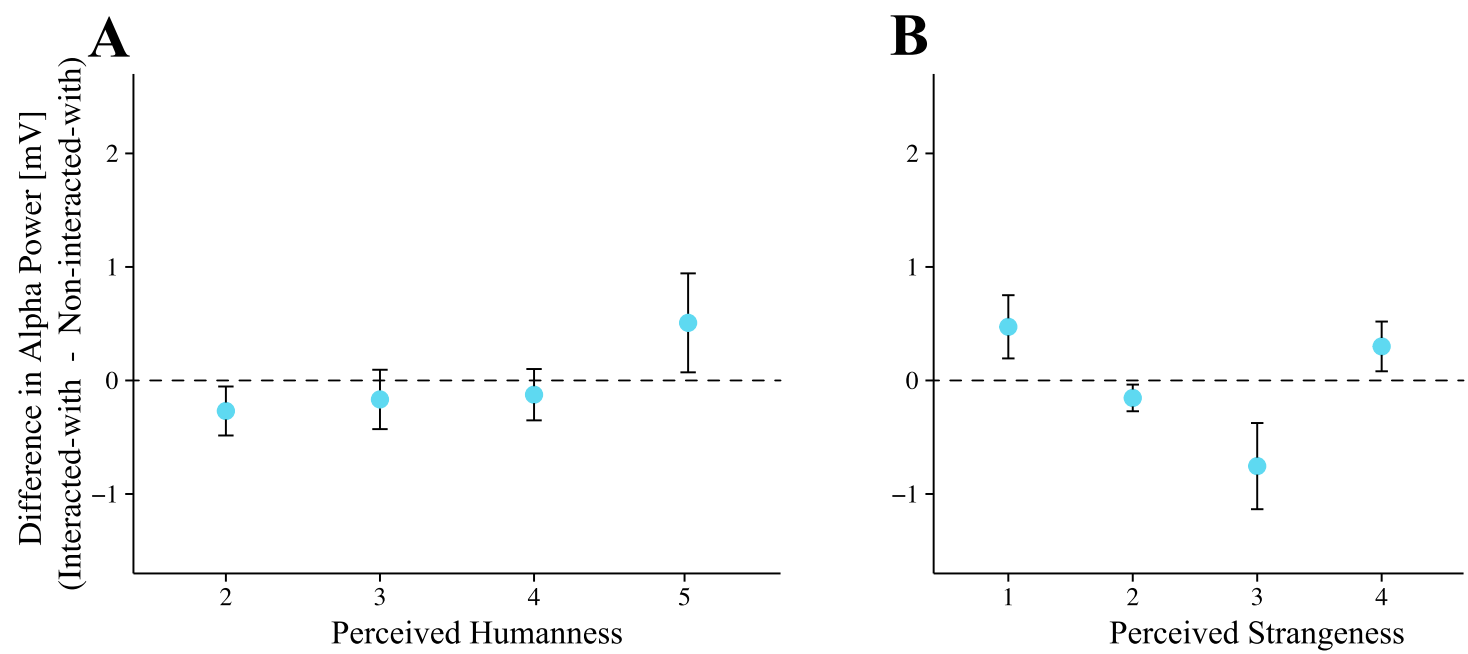

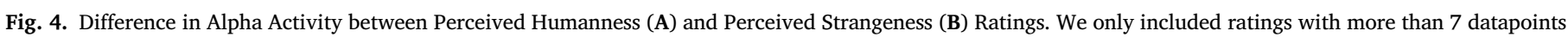

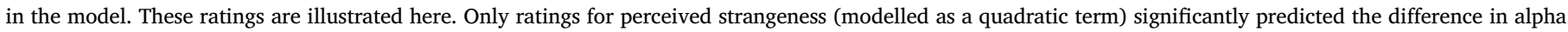
power to the presentation of the faces of the Interacted-with avatar compared to the Non-interacted-with avatar. Error bars represent standard error. 
where participants increasingly use their partners' grammar in their own utterances (Bock, 1986), and recent studies have shown that the magnitude with which participants adapt their language behaviour varies as a function of the participant's social opinion ratings of their partner. In our previous study (using the same avatars as in the current study), we observed an inverted U-shaped curve such that the maximal syntactic priming effect occurred for the middle ratings of perceived strangeness. To relate this to participant performance in the presence of a secondary individual and our attention hypothesis, when avatar interaction happens in concurrence with a task, such as syntactic priming, the participant has to divide attention between the faces and the task. Therefore, when little attention is given to the avatar itself (as with the medially-rated avatars), then more attention is available to complete the task, which would result in a better task performance (in this case, more syntactic priming) compared to doing the task in the presence of a secondary individual the participant finds extremely likeable or unlikeable. This is in line with the distraction-conflict hypothesis (Baron, 1986): The presence of others is a distraction, which leads to attentional conflict in terms of cognitive overload and selective focusing of attention.

To conclude, the first aim of our study was to determine whether attentional allocation varies when viewing faces of an individual the participant has interacted with and evaluated compared to not. Given the modulations in alpha activity observed, it is most likely that the capture of attention plays a role in modulating the behavioural effects seen in the social psychology literature. Our second aim was to determine whether this modulation of alpha activity (and therefore attention) differs as a function of rating and we show that it does. This clarifies why participant behaviour is different depending on whether they positively or negatively view the secondary individual, an effect seen not only in social psychology but also in psycholinguistics.

\section{Appendix A. Supplementary data}

Supplementary data related to this article can be found at https://doi. org/10.1016/j.neuroimage.2018.03.034.

\section{References}

Bates, D., Maechler, M., Bolker, B., 2012. lme4: Linear Mixed-effects Models Using S4 Classes (2011). R package version 0.999375-42.

Bond, C.F., Titus, L.J., 1983. Social facilitation: a meta-analysis of 241 studies. Psychol. Bull. 94 (2), 265-292.

Dan-glauser, E.S., Scherer, K.R., 2011. The Geneva affective picture database ( GAPED ): a new 730-picture database focusing on valence and normative significance. Behav. Res. Methods 43, 468-477. http://doi.org/10.3758/s13428-011-0064-1.

Delorme, A., Makeig, S., 2004. EEGLAB: an open source toolbox for analysis of single-trial EEG dynamics including independent component analysis. J. Neurosci. Methods 134 (1), 9-21. http://doi.org/10.1121/1.4929899.

Fox, J., Weisberg, S., 2011. Car: Companion to Applied Regression.

Foxe, J.J., Simpson, G.V., Ahlfors, S.P., 1998. Parieto-occipital- 1 0Hz activity reflects anticipatory state of visual attention mechanisms. Neuroreport 9 (17), 3929-3933.

Haegens, S., Nacher, V., Luna, R., Romo, R., Jensen, O., 2011. Alpha-oscillations in the monkey sensorimotor network influence discrimination performance by rhythmical inhibition of neuronal spiking. Proc. Natl. Acad. Sci. U. S. A. 108 (48), 19377-19382.

Hamel-Thibault, A., Thénault, F., Whittingstall, K., Bernier, P., 2015. Delta-band oscillations in motor regions predict hand selection for reaching. Cereb. Cortex 1-11.

Herman, C.P., 2015. The social facilitation of eating. A review. Appetite 86, 61-73. http://doi.org/10.1016/j.appet.2014.09.016.
Heyselaar, E., Hagoort, P., Segaert, K., 2015. In dialogue with an avatar, language behavior is identical to dialogue with a human partner. Behav. Res. Meth. 1-15. https://doi.org/10.3758/s13428-015-0688-7.

Heyselaar, E., Hagoort, P., Segaert, K., 2017. How social opinion influences syntactic processing - an investigation using Virtual Reality. PloS One 12 (4).

Kliegl, O., Pastötter, B., Bäuml, K.-H.T., 2015. The contribution of encoding and retrieval processes to proactive interference. J. Exp. Psychol. Learn. Mem. Cognition 41, 1778-1789.

Klimesch, W., 1997. EEG-alpha rhythms and memory processes. Int. J. Psychophysiol. 26 (1-3), 319-340. http://doi.org/10.1016/S0167-8760(97)00773-3.

Lange, J., Oostenveld, R., Fries, P., 2013. Reduced occipital alpha power indexes enhanced excitability rather than improved visual perception. J. Neurosci. 33 (7), 3212-3220.

Looser, C.E., Wheatley, T., 2010. The tipping point of animacy: how, when, and where we perceive life in a face. Psychol. Sci. 21 (12), 1854-1862. http://doi.org/10.1177/ 0956797610388044.

Lott, A.J., Lott, B.E., 1961. Group cohesiveness, communication level, and conformity. J. Abnorm. Soc. Psychol. 62 (6), 408.

Mandell, A.R., Smith, M.A., Martini, M.C., Shaw, T.H., Wiese, E., 2015. Does the presence of social agents improve cognitive performance on a vigilance task?. In: International Conference on Social Robotics. Springer International Publishing, pp. 421-430. http://doi.org/10.1007/978-3-319-25554-5.

Maris, E., Oostenveld, R., 2007. Nonparametric statistical testing of EEG- and MEG-data. J. Neurosci. Methods 164 (1), 177-190. http://doi.org/10.1016/j.jneumeth.2007.03. 024.

Mazaheri, A., Jensen, O., 2010. Rhythmic pulsing: linking ongoing brain activity with evoked responses. Front. Hum. Neurosci. 4.

Mazaheri, A., van Schouwenburg, M.R., Dimitrijevic, A., Denys, D., Cools, R., Jensen, O., 2014. Region-specific modulations in oscillatory alpha activity serve to facilitate processing in the visual and auditory modalities. NeuroImage 87, 356-362.

Menenti, L., Gierhan, S.M.E., Segaert, K., Hagoort, P., 2011. Shared language: overlap and segregation of the neuronal infrastructure for speaking and listening revealed by functional MRI. Psychol. Sci. J. Am. Psychol. Soc./APS 22 (9), 1173-1182. https:// doi.org/10.1177/0956797611418347.

Nass, C., Moon, Y., Carney, P., 1999. Are respondents polite to computers? Social desirability and direct responses to computers. J. Appl. Soc. Psychol. 29 (5), 1093-1110.

Oostenveld, R., Fries, P., Maris, E., Schoffelen, J.M., 2011. FieldTrip: open source software for advanced analysis of MEG, EEG, and invasive electrophysiological data. Comput. Intell. Neurosci. 2011. http://doi.org/10.1155/2011/156869.

R Core Development Team, 2011. R: a Language and Environment for Statistical Computing. Vienna, Austria.

Ringelmann, M., 1913. Recherches sur les moteurs animes: travail de l'homme [Research on animate sources of power: the work of man]. Ann. l'Institut Natl. Agron. XII, 1-40.

Segaert, K., Mazaheri, A., Hagoort, P., 2017. Binding language: structuring sentences through precisely timed oscillatory mechanisms. Eur. J. Neurosci.

Segaert, K., Menenti, L., Weber, K., Hagoort, P., 2011. A paradox of syntactic priming: why response tendencies show priming for passives, and response latencies show priming for actives. PloS One 6 (10). http://doi.org/10.1371/journal.pone.0024209.

Shahin, A., Pitt, M., 2012. Alpha activity marking word boundaries mediates speech segmentation. Eur. J. Neurosci. 36 (12), 3740-3748.

Siegel, M., Donner, T.H., Engel, A.K., 2012. Spectral fingerprints of large-scale neuronal interactions. Nat. Rev. Neurosci. 13 (February), 20-25. http://doi.org/10.1038/ nrn3137.

Strauss, B., 2002. Social facilitation in motor tasks: a review of research and theory. Psychol. Sport Exerc. 3 (3), 237-256.

van Diepen, R.M., Cohen, M.X., Denys, D., Mazaheri, A., 2015. Attention and temporal expectations modulate power, not phase, of ongoing alpha oscillations. J. Cognitive Neurosci. 27 (8), 1573-1586.

van Diepen, R.M., Mazaheri, A., 2017. Cross-sensory modulation of alpha oscillatory activity: suppression, idling and default resource allocation. Eur. J. Neurosci.

van Diepen, R.M., Miller, L.M., Mazaheri, A., Geng, J.J., 2016. The Role of Alpha Activity in Spatial and Feature-based Attention. ENeuro, 3(5), ENEURO-0204.

Weatherholtz, K., Campbell-Kibler, K., Jaeger, T.F., 2014. Socially-mediated syntactic alignment. Lang. Var. Change 26 (3), 387-420. http://doi.org/10.1017/ S0954394514000155.

Whittinghill, D.M., Ziegler, B., Moore, J., Case, T., 2015. Nasum virtualis: a simple technique for reducing simulator sickness in head mounted VR. In: Game Developers Conference. San Francisco. 